\title{
Optic Neuritis in Acute Neuroborreliosis (Lyme Disease) in the U.S.-Mexican Border: Case Report*
}

\author{
Ihtesham A. Qureshi1\#, Michael F. Maldonado², Gustavo J. Rodriguez, \\ Salvador Cruz-Flores' ${ }^{1}$, Alberto Maud ${ }^{1}$ \\ ${ }^{1}$ Neurology Department, Texas Tech University Health Sciences Center, EI Paso, USA \\ ${ }^{2}$ Ophthalmology Department, Texas Tech University Health Sciences Center, EI Paso, USA \\ Email: "drqureshi786@hotmail.com, Michael.f.maldonado@ttuhsc.edu,Gustavo.j.rodriguez@ttuhsc.edu, \\ alberto.maud@ttuhsc.edu, Salvador.cruz-flores@ttuhsc.edu
}

Received 2 March 2016; accepted 8 May 2016; published 11 May 2016

Copyright (C) 2016 by authors and Scientific Research Publishing Inc.

This work is licensed under the Creative Commons Attribution International License (CC BY).

http://creativecommons.org/licenses/by/4.0/

c) (i) Open Access

\section{Abstract}

Objective: We describe a patient diagnosed with acute neuroborreliosis presenting with anterior optic neuritis (papillitis) in a non-endemic region. Case Presentation: A 43-year-old previously healthy right handed man admitted due to an insidious onset of severe headache and spells of ascending paresthesias from his right foot into his right arm and face followed by speech arrest and clumsiness of his right hand. His neurologic exam was significant for somnolence, nuchal rigidity and Kernig and Brudzinski signs were present. MRI of the brain with gadolinium showed diffuse hyperintense signal involving the supra and infratentorial cortical sulci, with associated faint diffuse leptomeningeal enhancement, consistent most likely with diffuse leptomeningoencephalitis. EEG: normal. CSF VDRL was negative. Dilated fundus exam revealed mild optic nerve edema more significant to the left than to the right eye, confirmed and measured by spectral domain OCT (Optical Coherence Tomography). There was an evidence of posterior uveitis with an early vitreous hemorrhage superficial to the left optic nerve. Lyme disease serum antibody (IgM) Immunoblotting was positive in 2 bands confirming the diagnosis of neuroborreliosis. Conclusion: Optic nerve involvement in Lyme disease is an uncommon complication that should be confirmed by specific diagnostic criteria to establish its causal relation.

\section{Keywords}

Optic Neuritis, Lyme Disease, Non-Endemic, Papillitis

\footnotetext{
*All authors contributed equally (written, revised) before the manuscript was submitted.

\#Corresponding author.
} 


\section{Introduction}

Lyme disease (LD) is the most common arthropod-borne infectious disease in temperate regions of the northern hemisphere in Europe and is caused by Borrelia burgdorferi, a spirochete transmitted by Ixodes ticks [1]. The clinical manifestations of LD were first described in 1977 [2]. There is a wide variation of clinical presenting features of the disease that involves several organs like musculoskeletal, cardiovascular, peripheral and central nervous systems, as well as dermatological manifestations [3]. Along with this, a host of ophthalmological manifestations have also been described, ranging from chronic follicular conjunctivitis, keratitis [4] [5] optic neuritis to anterior, intermediate or posterior uveitis, scleritis and reversible horner's syndrome. These ocular manifestations are more frequently seen in late stages of the disease (2nd and 3rd stage). The first case of ocular lymeborreliosis was reported on 1985 where the patient went blind from severe panophthalmitis and Borrelia burgdorferi spirochetes were found in his vitreous. Despite a number of case reports claiming an association, strong evidence of a causal relationship has only been clearly established for papillitis [6]. There has been limited literature available regarding papillitis as an isolated ocular presentation of LD. We describe a patient diagnosed with acute neuroborreliosis presenting with anterior optic neuritis (papillitis) in a non-endemic region.

\section{Case Report}

A 43 years old previously healthy right-handed man was admitted due to an insidious onset of severe headache and spells of ascending paresthesias from his right foot into his right arm and face followed by speech arrest and clumsiness of the right hand. Two weeks prior was diagnosed of sinusitis and he received a course of antibiotics for a sore throat, nasal congestion and headaches. He presented afebrile and his general examination was unremarkable. His neurologic exam was significant for somnolence, nuchal rigidity and Kernig and Brudzinski signs were present. Chest X ray, Complete Blood Count (CBC), Comprehensive Metabolic Panel (CMP), Human Immunodeficiency Virus (HIV), Anti-Nuclear Antibodies (ANA), Rheumatoid Factor (RF), Anti-Neutrophil Cytoplasmic Antibody (ANCA), C3, C4, Thyroid Stimulating Hormone (TSH) and serum ammonia level were all within normal limits. Toxic and drug screen were negative.MRI of the Brain (FLAIR sequence) with gadolinium showed diffuse hyperintense signal involving the supra and infratentorial cortical sulci, with associated faint diffuse leptomeningeal enhancement, consistent with diffuse leptomeningoencephalitis (Figure 1). Electroencephalogram (EEG) was normal. CSF: opening pressure $10 \mathrm{~cm} \mathrm{H}_{2} \mathrm{O}$, a clear and colorless cerebrospinal fluid with 288 white cells (98\% lymphocytes), elevated proteins $(94 \mathrm{mg} / \mathrm{dl})$ and normal glucose. Gram stain showed no organism and bacterial, viral, fungal and tuberculosis cultures were negative. CSF VDRL was negative. He was discharged with diagnosis of undetermined (possible viral) meningitis. A week later he was readmitted to the hospital because increasing somnolence and disorientation in place and time. EEG showed severe bilateral generalized slow activity consistent with diffuse encephalopathy. He continued having repetitive short episodes of partial seizures in the right side of his body and transient episode of sinus bradycardia of 35 beats per minute. Transthoracic echocardiogram and cardiac enzymes were normal. He was loaded with antiepileptic drugs. A repeated brain MRI showed persistent diffuse supra and infratentorial leptomeningitis and subtle diffuse hyperintense signal on FLAIR and T2WI involving bilateral centrum semiovale and corona radiate consistent with encephalitis. Patient was treated with supportive management in the intensive care unit. The Lyme Antibody Screen in the serum came elevated (1.20 reference range: $\leq 0.90)$. Patient was started on oral Doxycycline $500 \mathrm{mg}$ twice a day and was discharge home. One week after the antibiotic treatment was started, he complained of ocular pain on the left eye with decrease in his visual acuity. He maintained 20/20 vision to the right and left eye and with only multiple visually disruptive peripheral photopsias and black spots temporal to left eye.

Ophthalmologic exam revealed normal anterior segment findings, reactive pupils, normal intraocular pressure and full extraocular muscle motility without restriction. Dilated fundus exam revealed mild optic nerve edema more significant in the left eye confirmed by spectral domain OCT (Optical Coherence Tomography). There was evidence of posterior uveitis with an early vitreous hemorrhage superficial to the left optic nerve (Figure 2). He was started on topical dose of Ketorolac Tromethamine $0.5 \%$ ophthalmic solution as prophylaxis for CME (Cystoid Macular Edema) and preservative free artificial tears to maintain corneal integrity. Lyme disease serum antibody (IgM) Immunoblotting was positive in 2 bands (23 kD and $39 \mathrm{kD}$ bands) confirming the diagnosis of acute neuroborreliosis. He completed a two-week course of oral Doxycycline and Ketorolac Tromethamine $0.5 \%$, preservative free artificial tears and 4 weeks later there was complete resolution of the vitreous hemorrhage and bilateral papillitis. Follow-up examination of the patient after 6 months revealed complete restoration 


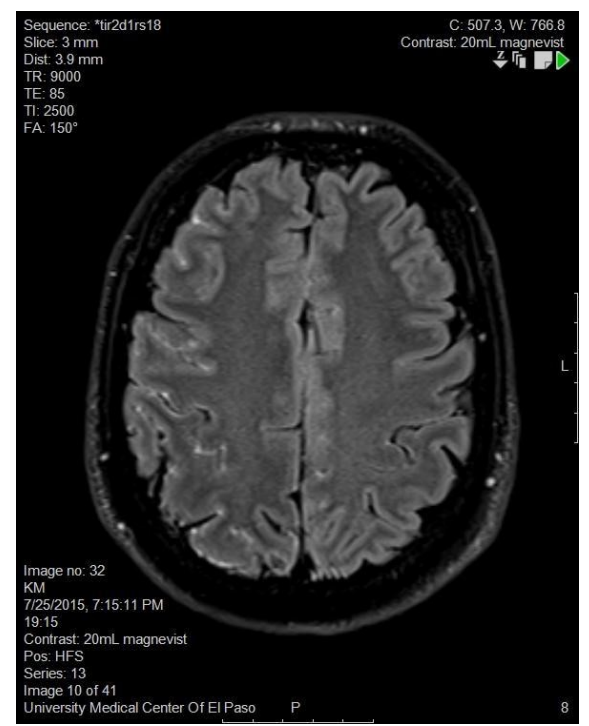

Figure 1. MRI Brain shows hyperintense signal involving cortical sulci and diffuse leptomeningeal enhancement.

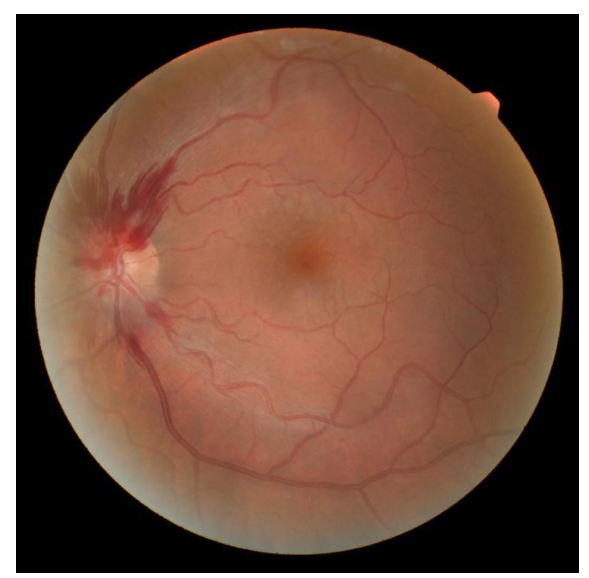

Figure 2. Left eye fundoscopy reveals small vitreous hemorrhage above the optic nerve with mild Optic Neuritis.

of the vision, no relapsing of the symptoms. Non-specific symptoms like mild headache and numbness have been reported. The left eye OCT scans after treatment revealed normal findings.

\section{Discussion}

There have been limited literature available that established a causal relationship of LD with Optic Neuritis (papillitis). The most pertinent documented case reports comprise a patient diagnosed with Lyme disease, initially presenting with a monocular papillitis, progressing to a bilateral papillitis several weeks later [7]. Another case in 1997, where unilateral papillitis was isolated as a clinical manifestation of acute Borrelia infection [8]. Additional case in 1996, where a patient with bilateral Lyme papillitis had been suffering with back pain for several months [9]. Two additional patients with presumed LD based on clinical findings presenting with papillitis have shown improvement following administration of intravenous methylprednisolone and ceftriaxone [10].

To confirm the diagnosis of neurologic LD with Optic Neuritis (papillitis), western blot is useful. Our presenting case has fulfilled the criteria for acute LD with positive western blot according to European Criteria (European Union Concerted Action on Lyme Borreliosis: EUCALB) and with strong evidence of a causal link 
between Optic Neuritis and LD, as described by Sibony et al. and Halperin et al. According to Sibony's recommendation [6], strong evidence of optic neuritis associated with active LD requires the following elements: Optic Neuritis, endemic exposure, negative VDRL, exclusion of multiple sclerosis and a positive serum titre (ELISA or Indirect fluorescent antibody), in association with one of the following: 1) Encephalitis or meningitis with CSF pleocytosis, intrathecal antibody production or CSF PCR positive for Borrelia burgdorferi DNA and a positive serum or CSF western blot; 2) recent signs of LD such as facial nerve palsy, arthritis or radiculo neuritis, with a positive serum ELISA confirmed by western blot; 3) recent physician diagnosed erythema migrans. Based on the Sibony recommendations, our case comes under Category II. There are only few reported cases of papillitis that appear to meet category II evidence for a causal link to LD.

In North America, Lyme Neuroborreliosis usually presents as lymphocytic meningitis with intermittent attacks of severe headache and mild meningism. As per the Centers for Disease Control and Prevention (CDC) criteria, a positive LD IgM immunoblot requires reactivity to 2 of 3 specific borrelial proteins that may indicate acute LD. However, if the IgM results obtained are positive after a month of onset of symptoms, likely represents a false positive IgM result rather than acute LD. In our case, the immunoblot test was performed on the 20th day after the onset of symptoms. It is recommended that antibiotic treatment should be initiated for patients who are seropositive with unexplained CSF pleocytosis, elevated CSF lyme antibody index or rising serum levels of Borrelia-specific antibodies, to avoid the late neurological consequences of chronic disease.

Our patient has shown astonishing results after treatment with antibiotics which can be clearly demonstrated by his neurologic, ophthalmologic and funduscopic recovery as well as the OCT (Optical Coherence Tomography) scans taken before the treatment and 2 weeks after. Our report represents a case of optic nerve involvement from acute LD in a non-endemic area. It highlights the importance of high suspicion for LD even in areas of presumed low incidence of the disease. The adherence to strict diagnostic criteria is particularly crucial in cases of acute LD presenting with atypical complications in non-endemic areas.

\section{Conclusion}

Optic nerve involvement in Lyme disease is an uncommon complication that should be confirmed by specific diagnostic criteria to establish its causal relation.

\section{Consent to Publish}

Informed consent from the patient was obtained.

\section{Ethics Approval}

Not applicable.

\section{Author's Contributions}

MFM, AM were involved in patient care and treatment; GJR, IAQ wrote the manuscript; SCF revised the manuscript.

\section{Availability of Data and Materials}

The dataset supporting the conclusions of this article is included within the article.

\section{Conflict of Interest}

None.

\section{References}

[1] Koedel, U., Fingerle, V. and Pfister, H.W. (2015) Lyme Neuroborreliosis-Epidemiology, Diagnosis and Management. Nature Reviews Neurology, 11, 446-456. http://dx.doi.org/10.1038/nrneurol.2015.121

[2] Steere, A.C., Malawista, S.E., Snydman, D.R., Shope, R.E., Andiman, W.A., Ross, M.R. and Steele, F.M. (1977) Lyme Arthritis: An Epidemic of Oligoarticular Arthritis in Children and Adults in Three Connecticut Communities. Arthritis \& Rheumatology, 20, 7-17. http://dx.doi.org/10.1002/art.1780200102 
[3] Centers for Disease Control and Prevention (CDC) (2007) Morbidity and Mortality Weekly Report, Vol. 56, Centers for Disease Control and Prevention (CDC), Lyme Disease-United States, 2003-2005, 573-576.

[4] Flach, A.J. and Lavoie, P.E. (1990) Episcleritis, Conjunctivitis and Keratitis as Ocular Manifestations of Lyme Disease. Ophthalmology, 97, 973-975. http://dx.doi.org/10.1016/S0161-6420(90)32474-0

[5] Mombaerts, I.M., Maudgal, P.C. and Knockaert, D.C. (1991) Bilateral Follicular Conjunctivitis as a Manifestation of Lyme Disease [Letter]. American Journal of Ophthalmology, 112, 96-97. http://dx.doi.org/10.1016/S0002-9394(14)76225-7

[6] Sibony, P., Halperin, J., Coyle, P.K. and Patel, K. (2005) Reactive Lyme Serology in Optic Neuritis. Journal of NeuroOphthalmology, 25, 71-82. http://dx.doi.org/10.1097/01.WNO.0000166060.35366.70

[7] Kienbacher, S., Muhlbauer-Ries, E. and Schmidt, B. (1996) Okulare Neuroborreliose-Nachweis Durch Polymerase Chain Reaction. Spektrum der Augenheilkunde, 10, 80-83. http://dx.doi.org/10.1007/BF03164182

[8] Pradella, S.P., Krause, A. and Muller, A. (1997) Acute Borrelia Infection. Unilateral Papillitis as Isolated Clinical Manifestation. Ophthalmologe, 94, 591-594. http://dx.doi.org/10.1007/s003470050165

[9] Gerard, P., Canaple, S. and Rosa, A. (1996) Meningopapillitis Disclosing Lyme Disease. Revue Neurologique (Paris), 152, 476-478.

[10] Amer, R., Brannan, S. and Forrester, J.V. (2009) Inflammatory Choroidalneovascular Membrane in Presumed Ocular Lyme Borreliosis. Acta Ophthalmologica, 87, 346-348. http://dx.doi.org/10.1111/j.1755-3768.2007.01160.x

\section{List of Abbreviations}

LD: Lyme Disease;

CBC: Complete Blood Count;

CMP: Comprehensive Metabolic Panel;

HIV: Human Immunodeficiency Virus;

ANA: Anti-Nuclear Antibodies;

RF: Rheumatoid Factor;

ANCA: Anti-Neutrophil Cytoplasmic Antibody;

TSH: Thyroid Stimulating Hormone;

EEG: Electroencephalogram;

OCT: Optical Coherence Tomography;

CME: Cystoid Macular Edema;

EUCALB: European Union Concerted Action on Lyme Borreliosis;

CDC: Centers for Disease Control and Prevention. 